\title{
O ECOSSISTEMA DE INOVAÇÃO DO DISTRITO FEDERAL BRASILEIRO E O PAPEL DA FAP-DF NO APOIO ÀS STARTUPS E NOVAS EMPRESAS DE BASE TECNOLÓGICA
}

\author{
Bruno Alencar Pereira ${ }^{1}$ \\ Josivania Silva Farias ${ }^{2}$
}

\begin{abstract}
This study identifies and systematizes the Distrito Federal (DF) innovation ecosystem, in Brazil, highlighting the role of the Fundação de Apoio à Pesquisa do Distrito Federal (FAP-DF) to the development of innovative ventures (startups and new technologybased firms). The study is based on qualitative documentary and descriptive research, considering the beneficial factors for development proposed by Stam and Van de Ven (2019). The results demonstrate a promising expansion of the DF ecosystem, and the role of FAP-DF evidenced in the institutional and resources contexts by facilitating the developing of these ventures through institutional support for the dissemination of innovation, resources for qualification in RD\&I and financial investments.
\end{abstract}

Keywords: ecosystem; innovation; entrepreneurship; startups; new technology-based firms.

Resumo: Este trabalho identifica e sistematiza o ecossistema de inovação do Distrito Federal destacando o papel da Fundação de Apoio à Pesquisa do Distrito Federal (FAP-DF) para o desenvolvimento de empreendimentos inovadores (startups e novas empresas de base tecnológica). O estudo fundamentanta-se em pesquisa qualitativa documental e descritiva, considerando os fatores benéficos de desenvolvimento propostos por Stame Van de Ven (2019). Os resultados demonstram a promissora expansão do ecossistema do DF, e especificamente o papel FAP-DF evidenciado nos contextos institucional $e$ de recursos facilitando o desenvolvimento destes empreendimentos por meio de apoio institucional para a disseminação da inovação, recursos para qualificação em PD\&I, e investimentos por subvenção econômica.

Palavras-chave: ecossistema; inovação; empreendedorismo; startups; novas empresas de base tecnológica.

\section{INTRODUÇÃO}

Mudanças estruturais no mercado potencializaram a ligação eficaz entre ciência, tecnologia e negócios fomentando a evolução de ecossistemas empresariais como tentativas

\footnotetext{
${ }^{1}$ Programa de Pós-Graduação em Administração (FACE/PPGA) na Universidade de Brasília (UnB). Brasília/DF - Brasil. E-mail: alenbruno@gmail.com.

${ }^{2}$ Programa de Pós-Graduação em Administração (FACE/PPGA) na Universidade de Brasília (UnB). Brasília/DF - Brasil. E-mail: josivania@unb.br.
} 
organizadas de estabelecer ambientes propícios ao êxito de empreendimentos recémestabelecidos (Singh \& Gaur, 2018; Audretsch et al., 2019).

Os ecossistemas, comumente, envolvem um conjunto de atores e fatores interdependentes que são governados de modo que possibilitam o empreendedorismo, fornecendo infraestrutura, apoio institucional e incentivos para o trabalho em rede para empresas estabelecidas ou novos empreendimentos tecnológicos, compondo assim a Tríplice Hélice da inovação (Martin-Rios \& Erhardt, 2017; FAP-DF, 2019; Stam \& Van de Ven, 2019).

O modelo de inovação em Tríplice Hélice disseminado por Etzkowitz e Leydesdorff (1995) refere-se a um conjunto de interações entre universidade, indústria e governo, para promover o desenvolvimento econômico e social, onde cada estrutura helicoidal da inovação é representada com suas respectivas interações e sobreposições. Embora este trabalho se baseie na abordagem seminal da Tríplice Hélice, ressaltamos a existência e importância de modelos derivados que agregaram outros elementos, como a Quádrupla Hélice (universidade, indústria, governo e sociedade) (Carayannis \& Campbell, 2009) e, mais recentemente, a Quíntupla Hélice que agrega ambientes naturais da sociedade e da economia (Carayannis, Barth \& Campbell 2012).

Poucos estudos consideram a dinâmica do desenvolvimento de startups e novas empresas de base tecnológica no contexto de ecossistemas ou como podem cocriar valor para gerar inovações (Parida \& Wincent, 2019; Sjödin, 2019). Neste sentido, o objetivo deste estudo foi identificar e sistematizar o atual ecossistema de inovação do Distrito Federal destacando o papel da Fundação de Apoio à Pesquisa do Distrito Federal (FAP-DF) no suporte e desenvolvimento de empreendimentos inovadores, como um ator fundamental no contexto da Tríplice Hélice para a criação da inovação.

\section{REVISÃO DA LITERATURA}

\subsection{ECOSSISTEMAS DE INOVAÇÃO}

A literatura fornece amplo arcabouço teórico sobre inovação e ecossistemas (Sjödin, 2019), em que ecossistemas de inovação se constituem como sistemas de longo prazo ou temporários, formado por vários atores e organizações, cujo objetivo funcional é promover a interação, a comunicação e o desenvolvimento de tecnologias, inspirando a inovação dentro da 
rede (Wu et al., 2017; Skute et al., 2019). Ecossistemas de inovação têm papel relevante no desempenho das empresas, especialmente em seu estágio inicial (Monteiro, 2019). As redes interorganizacionais, nestes ecossistemas, podem garantir recursos valiosos como conexões, mais investimetnos e capacidades para empreendimentos inovadores (Parida \& Wincent, 2019).

Ecossistemas vibrantes, como o Vale do Silício (EUA), Distrito da inovação e projeto @22 Barcelona (Espanha), região de Zhongguancun (China), Israel a 'nação startup', e ambientes nacionais como o San Pedro Valley em Belo Horizonte/MG, Porto Digital em Recife/PE e Florianópolis/SC, estimulam o aumento de conhecimento e recursos, gerando resultados bem-sucedidos de inovação para dinamicidade e crescimento da economia regional.

\subsection{FATORES FACILITADORES DE DESENVOLVIMENTO DE NOVOS EMPREENDIMENTOS POR MEIO DE ECOSSISTEMAS DE INOVAÇÃO}

O ecossistema inovador tem papel relevante por meio de fatores que impactam no desempenho das empresas, especialmente em seu estágio inicial, pois estas demandam recursos que sustentem seu crescimento (Bhawe et al., 2017; Monteiro, 2019; Stam \& Van de Ven, 2019). Nesse contexto, para a análise das evidências empíricas deste estudo, sobre o ecossistema do Distrito Federal, em especial da FAP-DF, serão considerados os conceitos adotados no framework de fatores benéficos para ecossistema empreendedor proposto por Stam e Van de Ven (2019), demonstrado na Tabela 1.

Tabela 1 - Fatores de fomento ao ecossistema de empreendedorismo e negócios inovadores

\begin{tabular}{lll}
\hline \multicolumn{1}{c}{ Contexto } & \multicolumn{1}{c}{ Fator } & \multicolumn{1}{c}{ Definição } \\
\hline \multirow{2}{*}{ Institucional } & Formalidade & $\begin{array}{l}\text { As regras e governança estabelecidas entre os atores do ecossistema } \\
\text { por meio de mecanismos formais. }\end{array}$ \\
\cline { 2 - 3 } & Cultura & $\begin{array}{l}\text { Contexto cultural das instituições que fomentam a disseminação do } \\
\text { empreendedorismo inovador e a criação de novas empresas. }\end{array}$ \\
\cline { 2 - 3 } & $\begin{array}{l}\text { Network } \\
\text { Recursos físicos e } \\
\text { infraestrutura }\end{array}$ & $\begin{array}{l}\text { Contexto de ações e capital social que possibilitam a conectividade } \\
\text { entre os atores e envolvimento de colaborações em prol da } \\
\text { inovação. }\end{array}$ \\
\cline { 2 - 3 } Recurspos & $\begin{array}{l}\text { Conhecimento. } \\
\text { financeiros }\end{array}$ & $\begin{array}{l}\text { A presença de meios financeiros para investir em atividades de } \\
\text { PD\&I. }\end{array}$ \\
\cline { 2 - 3 } & Liderança & $\begin{array}{l}\text { Liderança que provê direcionamentos coletivos para ações } \\
\text { relacionadas à inovação. }\end{array}$ \\
\cline { 2 - 3 } & Capital humano & As habilidades, conhecimento e experiência de talentos envolvidos. \\
\cline { 2 - 3 } & Conhecimento & $\begin{array}{l}\text { Investimento em ciência e tecnologia para a criação de } \\
\text { conhecimento e inovação. }\end{array}$ \\
\hline Fonte: Adaptado de
\end{tabular}


A seguir, são demonstrados os métodos adotados no trabalho, o ecossistema do Distrito Federal, a atuação dos atores envolvidos segmentados na perspectiva da Tríplice Hélice, e a discussão sobre o papel da FAP-DF no desenvolvimento de startups e novas empresas de base tecnológica.

\section{PROCEDIMENTOS METODOLÓGICOS ADOTADOS NO ESTUDO}

Para a elaboração e demonstração do ecossistema de inovação do Distrito Federal, como caso de estudo fundamentado na concepção da Tríplice Hélice, adotou-se abordagem qualitativa com análise documental descritiva, por meio de relatórios, sites, censos e demais publicações. No total, foram submetidos à análise documental descritiva 11 documentos, entre oficiais, notícias, normas/leis etc.

Para a análise e discussão do papel da FAP-DF, unidade específica de análise do caso estudado, no apoio do desenvolvimento de startups e novas empresas de base tecnológica, utilizaram-se na análise de conteúdo, como categorias temáticas estabelecidas a priori, os fatores benéficos para ecossistemas de empreendedorismo inovador do framework de Stam e Van de Ven (2019). Foram observados os contextos e evidências documentais que se alinhavam aos fatores propostos do framework. Ressalta-se, que na construção do ecossistema de inovação do DF não foram integradas organizações que não estivessem espacialmente situadas no Distrito Federal.

\section{O ECOSSISTEMA DE INOVAÇÃO DO DISTRITO FEDERAL}

Em 2019, o Brasil se posicionou como $64^{\circ}$ colocado no ranking mundial de inovação, ficando atrás de países em desenvolvimento como Chile $\left(47^{\circ}\right)$, México $\left(56^{\circ}\right)$, Índia $\left(57^{\circ}\right)$ e África do Sul $\left(58^{\circ}\right)$. Embora o país não esteja na fronteira tecnológica para inovação, o Distrito Federal destacou-se nos quesitos 'publicação científica' e 'infraestrutura de telecomunicações', em cujas categorias o DF está em $1^{\circ}$ lugar no País (FAP-DF, 2019). A integração de ciência, infraestrutura, talentos e boas políticas de fomento à inovação possibilitam que o Distrito Federal seja terreno fértil para empreendimentos inovadores.

Essa promissora ambientação regional para a inovação se remete ao arcabouço de leis 
que possibilitaram iniciativas mais promissoras no contexto da Tríplice Hélice, iniciando-se em contexto nacional com a Lei número 10.973 de dezembro de 2004, que balizou o fomento a inovação no Brasil, revogada pelo Decreto 9.283, de 2018. Regionalmente, ressalta-se o Projeto de Lei da Inovação $n^{\circ} 1.536$, de 2017, pelo qual o DF se torna a primeira unidade da Federação a ter uma lei local de inovação, somando-se à Política Distrital de Ciência, Tecnologia e Inovação - Inova Brasília, instituída pelo Decreto n 38.126 de 2017, e mais recente pela criação do Parque Tecnológico BioTIC.

De acordo com a ABSStartups (2019), se tratando de empreendimentos inovadores, especificamente, a comunidade de startups de Brasília é a maior da região Centro-Oeste em número de startups, sendo ao todo 209 startups ativas. Este cenário promissor para empreendimentos inovadores se relaciona com a evolução do ecossistema do Distrito Federal, envolvendo ambientes de inovação, conhecimento tecnológico das universidades e instituições de tecnologia, negócios inovadores, investimentos públicos e privados, bem como políticas e programas de fomento governamentais, constituindo o ecossistema de inovação do Distrito Federal baseado na abordagem da Tríplice Hélice, conforme demonstrado pela Figura 1.

Figura 1 - O ecossistema de inovação do Distrito Federal abordado pela lógica da Tríplice Hélice

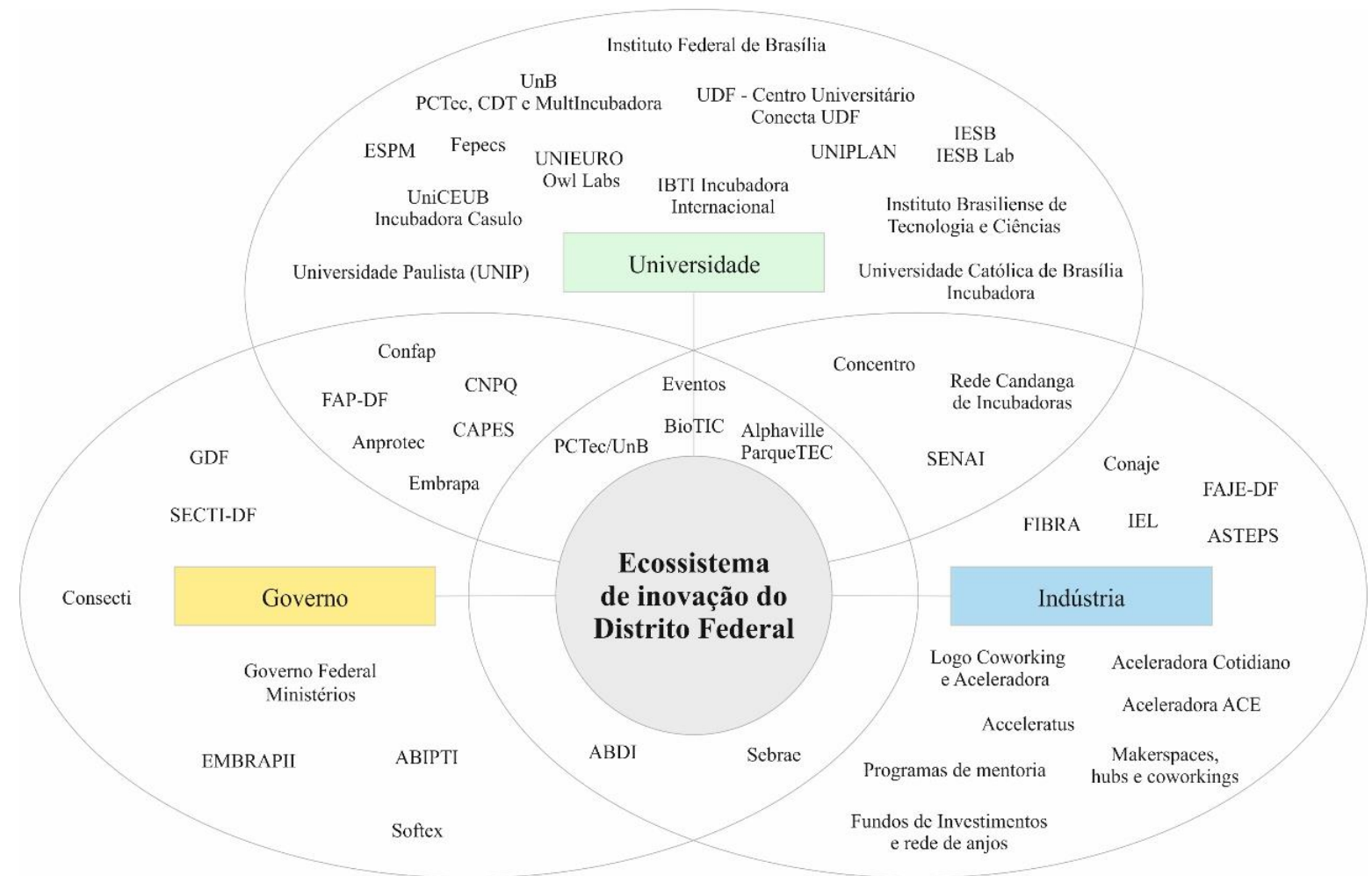

Fonte: Adaptado de Pereira et al. (2018). 


\subsection{UNIVERSIDADE}

A Universidade é representada pelas 13 instituições identificadas na Figura 1 que constituem a hélice do conhecimento, promovendo ciência básica e aplicada, formação qualificada e desenvolvimento de capacidades científicas e tecnológicas absorvidas pela comunidade acadêmica, se estendendo para a sociedade. E, de forma mais enfática, por meio de projetos de PD\&I, incubadoras de empresas, Núcleos de Inovação Tecnológica (NITs), hubs e centros de empreendedorismo, empresas juniores, que reduzem o gap existente na relação universidade-empresa em interação com políticas públicas governamentais, programas de fomento e ações interconectadas. Nas universidades, somam-se inúmeras ações direcionadas ao empreendedorismo e inovação como eventos de CT\&I, em grande parte apoiados pela FAP-DF, como hackatons; meet-ups; desafios inovadores; olimpíadas de empreendedorismo etc.

\subsection{INDÚSTRIA}

A Indústria representa o setor produtivo que é força motora para o desenvolvimento econômico regional, responsável pela difusão da inovação na conversão da ideação e desenvolvimento de produtos ou serviços em valor econômico e social. Empresas, associações, comunidades e mecanismos de apoio citados na Figura 1 constituem o conjunto de atores desta hélice. Integrando o Sistema S, destaca-se a atuação do Serviço Brasileiro de Apoio às Micro e Pequenas Empresas (Sebrae); a Federação das Indústrias do DF (FIBRA); o Instituto EuvaldoLodi (IEL); e o Serviço Nacional de Aprendizagem Industrial (SENAI).

Incubadoras e aceleradoras são mecanismos de apoio ao desenvolvimento de empreendimentos inovadores com forte ênfase em criatividade e inovação. No Distrito Federal, destacam-se incubadoras apoiadas pela Rede Candanga de Incubadoras como a MultIncubadora (CDT/UnB), Casulo (UniCEUB), IBTI Incubadora Internacional, Conecta UDF, Incubadora da UCB; as aceleradoras Cotidiano, ACE Brasília, Logo Coworking e Aceleradora, Aceleradora Acceleratus, e o Oops Programa de Pré-Aceleração do UDF. Makerspaces, hubs e coworkings são outros espaços compartilhados e criativos que se alinham aos ambientes de inovação. Programas de mentoria suportam as capacidades empreendedoras e absortivas dos empreendimentos. E, se tratando de investimentos, tem-se o aumento da presença de redes que conectam investidores a fundadores e fundos de investimentos, bem como investidores anjos 
com conhecimento técnico, agregando smartmoney para empreendimentos em estágio inicial.

\subsection{GOVERNO}

Pelo fato de o Distrito Federal ser a capital do Brasil, diversas organizações governamentais estão presentes no DF. Portanto, é imprescindível integrar as ações do Governo Federal ao ecossistema de inovação local. No âmbito do Governo Federal se destaca o Ministério da Ciência, Tecnologia, Inovações e Comunicações (MCTIC) desenvolvendo ações de promoção da inovação e empreendedorismo inovador por meio de programas de ampla difusão. Vinculado ao MCTIC, acrescenta-se o Conselho Nacional de Desenvolvimento Científico e Tecnológico (CNPq) e também a Coordenação de Aperfeiçoamento de Pessoal de Nível Superior (CAPES) vinculada Ministério da Educação (MEC), ambas com atribuição de fomentar a pesquisa científica, tecnológica e de inovação, e a atuação de pesquisadores em empresas.

Outras associações promovendo parcerias com o MCTIC reforçam o arcabouço de apoio ao desenvolvimento tecnológico e empreendedorismo inovador no país como a Empresa Brasileira de Pesquisa e Inovação Industrial (EMBRAPII); a Associação Nacional de Entidades Promotoras de Empreendimentos Inovadores (Anprotec); a Associação Brasileira das Instituições de Pesquisa Tecnológica e Inovação (ABIPTI); o Conselho Nacional das Fundações Estaduais de Amparo à Pesquisa (CONFAP); o Conselho Nacional de Secretários para Assuntos de Ciência, Tecnologia e Inovação (ConSECTI); e a Softex. Pelo Ministério da Agricultura, Pecuária e Abastecimento, destaca-se a Empresa Brasileira de Pesquisa Agropecuária (Embrapa) e pelo Ministério da Economia destaca-se a ligação com a Agência Brasileira de Desenvolvimento Industrial (ABDI).

Em âmbito regional, o Governo do Distrito Federal (GDF) alinha-se a este contexto desenvolvendo iniciativas locais por meio da Secretaria de Ciência, Tecnologia e Inovação (SECTI), sendo essencialmente executadas pela Fundação de Apoio à Pesquisa do Distrito Federal (FAP-DF). Como principal lócus deste estudo e pelas relevantes ações a seguir elencadas, a FAP-DF recebe destaque na próxima seção, na qual, além de promover a interação entre as três hélices (universidade, indústria e governo), destaca-se e analisa-se seu papel no desenvolvimento de startups e novas empresas de base tecnológica.

A FAP-DF é uma entidade com personalidade jurídica de direito privado, sob a forma 
de fundação pública. Sua missão é estimular, apoiar e promover o desenvolvimento científico, tecnológico e de inovação do Distrito Federal. A Fundação foi criada pela Lei No 347, de 04/11/1992, e implementada em 04 de novembro de 1993. Atualmente, a Fundação é vinculada à Secretaria de Ciência, Tecnologia e Inovação do Distrito Federal (SECTI), (FAP-DF, 2020). O apoio da FAP-DF na expansão do ecossistema local de inovação tem sido consistente por meio de amplo espectro envolvendo desde a oferta de bolsas de desenvolvimento tecnológico e inovação, apoio a universidades e institutos de tecnologia, eventos científicos e tecnológicos até o apoio direto para o desenvolvimento de soluções tecnológicas criadas por empreendimentos inovadores promovendo links com a academia (Díez-Vial \& MontoroSánchez, 2016).

A interação entre atores da Tríplice Hélice torna-se mais evidente pela existência dos parques tecnológicos ou científicos e tecnológicos. O Distrito Federal possui o Parque Tecnológico de Brasília (BioTIC), já implantado, e o Parque Tecnológico Alphaville, em implantação, e o Parque Científico e Tecnológico da Universidade de Brasília (PCTec/UnB).

O Parque Tecnológico de Brasília (BioTIC) foi criado para oferecer um ecossistema de cooperação e geração de negócios entre empreendedores, empresas, universidades e centros de pesquisa (BioTIC, 2020). Pela sua relevância para o ecossistema do DF, instituições como a FAP-DF, ABIPTI, SEBRAE, Anprotec, Embrapa, Instituto Federal Brasília (IFB), e a Fundação Universidade Aberta do Distrito Federal (Funab), possuem sede ou núcleos instalados no Parque.

Situado dentro do Campus Universitário Darcy Ribeiro, o Parque Científico e Tecnológico da Universidade de Brasília (PCTec/UnB) é responsável por estimular a interação entre empresas, governo e a comunidade científica, fomentando parcerias com instituições públicas e privadas, nacionais e internacionais com foco em PD\&I (PCTec, 2020).

O Parque Tecnológico Alphaville, ainda em processo de implantação, possui as seguintes empresas que já confirmaram a instalação de núcleos no local: UCB, Rede Brasileira de Cidades Inteligentes e Humanas, Iland Datacenter, Sebrae GO e DF, FAP-DF e Fapeg-GO, Espaço Multiplicidade, Escritório Colaborativo, ASTEPS e Cotidiano Aceleradora (Cidade Ocidental, 2020).

Depois de uma análise regional mais ampla do DF, a seção 5, a seguir, faz um recorte do ecossistema de inovação do Distrito Federal, em que se aprofundará a análise do papel da 
FAP-DF no desenvolvimento de startups e novas empresas de base tecnológica.

\section{O PAPEL DA FAP-DF NO APOIO AO DESENVOLVIMENTO DE STARTUPS E NOVAS EMPRESAS DE BASE TECNOLÓGICA}

Como lócus escolhido para este estudo, e pelas ações realizadas, a FAP-DF destaca-se no ecossistema de inovação do Distrito Federal por promover a interação entre as três hélices (universidade, indústria e governo), além de seu papel no desenvolvimento de startups e novas empresas de base tecnológica do DF. Para essa análise e discussão, como informado antes, foram relacionados os fatores benéficos para o desenvolvimento de ecossistemas de empreendedorismo inovador propostos por Stam e Van de Ven (2019) e as evidências empíricas documentais demonstradas na Tabela 2.

\subsection{CONTEXTO INSTITUCIONAL}

Quanto ao fator formalidade, a FAP-DF possui diversas parcerias por meio de contratos, convênios, termos de cooperação técnica, termos de cessão de bolsas e editais que estabelecem laços formais de atuação. Por se tratar de uma instituição pública, com dotação orçamentária regulamentada e auditada, se faz primordial a adoção de medidas transparentes e de accountability durante o processo de execução, além da instituição do Comitê Interno de Governança (CIG), que define a estratégia para celebração de parcerias com a FAPD-DF (FAPDF, 2019).

No que diz respeito ao fator cultura, pode-se observar a atuação da FAP-DF na difusão de cultura empreendedora através de eventos e atividades que disseminam o potencial da inovação, viabilizando cases de sucesso que estimulem o surgimento de novos empreendimentos. De acordo com o mapeamento da ABStartups (2019), verifica-se o crescimento de empresas inovadoras, pois a comunidade de empreendedorismo inovador da capital é a maior do Centro-Oeste, passando de 20 startups ativas em 2012 para 209 em 2019 (Whow, 2020).

Tratando-se do fator network, a FAP-DF articula movimentos pró-inovação estabelecendo nós e conexões que potencializam o capital social dos empreendimentos inovadores e apoiando iniciativas de interação entre os atores como eventos de CT\&I, projetos 
de cooperação técnico-científicos, e editais que articulam empresas e instituições de desenvolvimento tecnológico para potencializar as capacidades de inovação dos empreendimentos apoiados.

\subsection{CONTEXTO DE RECURSOS}

Em relação ao fator recursos fisicos e infraestrutura, pode-se analisar a atuação da FAPDF por duas perspectivas. Primeiro, quanto à sua localização espacial no Distrito Federal, com acesso aos demais atores, em que a FAP-DF atua em nova sede situada Parque Tecnológico BioTIC desde 2018. A FAP-DF assume posição estratégica, visto que importantes atores como a ABPITI, SEBRAE, Anprotec, Embrapa, Instituto Federal Brasília (IFB), e a Fundação Universidade Aberta do Distrito Federal (Funab) também se encontram instaladas no BioTIC, com a estimativa de concentrar cerca de 1,2 mil empresas dos ramos da tecnologia da informação e comunicação e da biotecnologia (GDF, 2020). Segundo, na perspectiva de acesso a recursos e infraestrutura, a FAP-DF provê por meio de parcerias e termos de cooperação com instituições tecnológicas, o acesso ao conhecimento, laboratórios e tecnologias que podem ser acessadas pelas empresas por meio de mecanismos apropriados de cooperação. Recursos de infraestrutura e para desenvolvimento tecnológico também são providos pelos diversos editais de apoio ao desenvolvimento de empreendimentos inovadores.

Quanto ao fator recursos financeiros, a FAP-DF oferece mecanismos específicos de fomento para o desenvolvimento de soluções tecnológicas conjuntamente com os recursos de obtenção de infraestrutura e insumos por meio de editais de subvenção econômica. Ressalta-se que a FAP-DF não atua com investimentos financeiros aplicados a empreendimentos na forma de equity. Entretanto, os recursos disponibilizados podem ser diretamente utilizados para a obtenção de materiais permanentes ou de consumo visando o desenvolvimento de soluções inovadoras pelos empreendimentos contemplados.

Quanto ao fator liderança, percebe-se a atuação da FAP-DF apoiando agentes ou evangelistas da inovação, como líderes do movimento no ecossistema regional constituído por fundadores de startups, mentores, consultores, e gestores de ambientes de inovação, que frequentemente atuam em atividades e eventos relacionados ao empreendedorismo inovador (ABStartups, 2019). A FAP-DF possibilita que gestores e líderes do movimento em suas instituições recebam condições viáveis para disseminação e fortalecimento das ações de 
inovação em suas comunidades.

Concernente ao fator capital humano, a presença de bons talentos é essencial para a criação e manutenção de um forte ecossistema empreendedor, cujo investimento em capital humano cria e retém força de trabalho, favorecendo a inovação (ABStartups, 2019). Aqui se analisa o relevante papel da FAP-DF na formação qualificada de talentos, provendo bolsas de pesquisa, iniciação científica e desenvolvimento tecnológico, principalmente para programas stricto sensu nas universidades ou por meio de programas e fomento a eventos de capacitação e de disseminação de CT\&I em articulação com universidades, institutos e outros atores.

Por último, em relação ao fator conhecimento, a FAP-DF investe recursos financeiros para desenvolvimento tecnológico e PD\&I por meio de diversos mecanismos como acordos de cooperação técnica, pesquisas de demanda espontânea e inovação em empreendimentos emergentes focados em tecnologia.

A Tabela 2, a seguir, esboça a síntese de fatores e evidências do papel da FAP-DF no desenvolvimento de startups e novas empresas de base tecnológica, a partir dos fatores propostos por Stam e Van de Ven (2019).

Tabela 2 - Síntese de fatores e evidências do papel da FAP-DF no desenvolvimento de startups e novas empresas de base tecnológica.

\begin{tabular}{|c|c|c|}
\hline Contexto & Fator & Evidência empírica documental analisada \\
\hline \multirow{3}{*}{ Institucional } & Formalidade & $\begin{array}{l}\text { Editais para inovação em microempresas e empresas de pequeno porte; } \\
\text { Edital Startups Inova Brasília; Seleção de propostas para apoio financeiro na } \\
\text { modalidade subvenção econômica à startups; Convênio n }{ }^{\circ} .03 / 2019 \text { com o } \\
\text { Senai para execução do projeto "DF mais Produtivo"; Convênio n }{ }^{\circ} .04 / 2019 \\
\text { em parceria com o Senai para qualificar profissionais em novas tecnologias } \\
\text { e empresas focadas na Indústria 4.0; Comitê Interno de Governança (CIG). }\end{array}$ \\
\hline & Cultura & $\begin{array}{l}\text { Editais de seleção pública de propostas de apoio à promoção, realização e } \\
\text { organização de eventos científicos, tecnológicos e de inovação; } \\
\text { Chamamentos públicos para execução de projetos de educação inovadora; e } \\
\text { Seleção pública de propostas para patrocínio. }\end{array}$ \\
\hline & Network & $\begin{array}{l}\text { Programa de tecnologia e inovação envolvendo os editais para apoio ao } \\
\text { desenvolvimento e inovação para micro e pequenas empresas e para } \\
\text { empresas emergentes de base tecnológica; Programa de difusão científica } \\
\text { com seleção pública de propostas de ações de popularização da ciência, } \\
\text { tecnologia e inovação no DF; Feira de negócios e inovação promovida pelo } \\
\text { Parque Científico e Tecnológico (PCTec) e Centro de Apoio ao } \\
\text { Desenvolvimento Científico e Tecnológico (CDT) da UnB; Campus Party. }\end{array}$ \\
\hline \multirow[t]{2}{*}{ Recursos } & $\begin{array}{l}\text { Recursos } \\
\text { físicos e } \\
\text { infraestrutura }\end{array}$ & $\begin{array}{l}\text { Mapa estrutural e localização do BioTIC disponibilizado pelo site } \\
\text { BioTIC/SA (BioTIC, 2020); Editais de START BSB de apoio financeiro na } \\
\text { modalidade subvenção econômica à startups/projetos inovadores; o } \\
\text { Programa Centelha; e Editais de apoio ao desenvolvimento tecnológico e de } \\
\text { inovação em empresas emergentes de base tecnológica. }\end{array}$ \\
\hline & Recursos & Edital para microempresas e empresas de pequeno porte; Edital Startups \\
\hline
\end{tabular}




\begin{tabular}{|c|c|}
\hline financeiros & $\begin{array}{l}\text { Inova Brasília; Edital de seleção pública de propostas para apoio financeiro } \\
\text { na modalidade subvenção econômica à startups. }\end{array}$ \\
\hline Liderança & $\begin{array}{l}\text { Editais de aquecimento do ecossistema de inovação do Distrito Federal; a } \\
\text { disponibilidade de recursos e apoio institucional por meio de Editais de } \\
\text { seleção pública de propostas de apoio à promoção, realização e organização } \\
\text { de eventos científicos, tecnológicos e de inovação. }\end{array}$ \\
\hline $\begin{array}{l}\text { Capital } \\
\text { humano }\end{array}$ & $\begin{array}{l}\text { Bolsas de iniciação científica, mestrado e doutorado; Convênio n }{ }^{\circ} .04 / 2019 \\
\text { em parceria com o Senai para qualificar profissionais em novas tecnologias } \\
\text { e empresas focadas na Indústria 4.0; e Chamamentos públicos para execução } \\
\text { de projetos de educação inovadora. }\end{array}$ \\
\hline Conhecimento & $\begin{array}{l}\text { Acordo de Cooperação Técnica (ACT) com a Embrapii, que consiste no } \\
\text { financiamento de projetos de inovação de demandas das empresas com } \\
\text { centros de pesquisa; Editais Startup Inova Brasília e Pesquisa e inovação em } \\
\text { microempresas e empresas de pequeno porte; e Seleção pública para apoio } \\
\text { financeiro ao desenvolvimento de produtos e processos inovadores. }\end{array}$ \\
\hline
\end{tabular}

Fonte: Elaboração da autoria (2020)

Como se verificou nesta seção, o framework de análise de Stam e Van de Ven (2019) subsidiou enormemente a análise dos mecanismos utilizados em ecossistemas de inovação, e todos os componentes/fatores benéficos propostos pelos autores foram verificados no ecossistema aqui estudado, demonstrando sua pertinência para esse tipo de estudo.

\section{CONCLUSÃO}

Este estudo identificou e sistematizou o ecossistema de inovação do Distrito Federal, explorando ações e dinâmicas que estendem o conhecimento sobre ecossistemas regionais de inovação, desdobrando sob quais condições empreendimentos inovadores podem obter oportunidades de absorção de conhecimento, capacidades e recursos para inovar. Destaca-se o ecossistema de inovação do Distrito Federal, percebido como um ecossistema em expansão e promissor e, especificamente, o papel da FAP-DF no desenvolvimento para a inovação promovendo suporte em contexto institucional e de recursos, evidenciando a ocorrência de fatores benéficos para o empreendedorismo inovador apresentado pelo framework proposto por Stam e Van de Ven (2019). Tais evidências são identificadas pela interação com os demais atores, especialmente direcionando apoio institucional para a disseminação da inovação, e provendo recursos para qualificação em PD\&I e investimentos por subvenção econômica a startups e novas empresas de base tecnológica.

A pesquisa abre novas perspectivas de investigação sobre ecossistemas de inovação para o desenvolvimento de empreendimentos inovadores. Uma possível contribuição teórica se relaciona com a possibilidade de generalização do framework utilizado para demais 
ecossistemas em âmbito regional e nacional, não somente destacando a atuação das fundações de amparo à pesquisa, mas também a atuação interconectada dos atores envolvidos. Ressaltase, ainda, a identificação de que os fatores propostos por Stam e Van de Ven (2019) se relacionam diretamente com abordagens teóricas da capacidade absortiva (ACAP), possibilitando insights para futuros estudos que relacionem tais fatores com este construto (Cohen \& Levintal, 1990; Pereira \& Farias, 2018).

Limitações do estudo sobre a concepção do ecossistema de inovação do Distrito Federal estão relacionadas ao fato de a pesquisa documental não ter sido realizada sem triangulação com outras evidências. Possivelmente, futuras pesquisas com triangulação de métodos, fontes, abordagens etc. poderão incluir a experiência e percepção de atores envolvidos para agregar outros elementos e ações não identificados neste trabalho. Da mesma forma, o estudo se ateve à discussão do ecossistema de inovação para o desenvolvimento de empreendimentos inovadores utilizando-se de recorte específico sobre o papel da FAP-DF. Estudos mais abrangentes poderiam envolver demais atores, ampliando o entendimento do suporte institucional e de recursos envolvidos para o desenvolvimento desses empreendimentos. Por fim, este estudo se limitou a fatores e ocorrência de ações realizadas pelo ecossistema, não se estendendo aos outputs para criação de valor existente no framework de Stan e Van de Ven (2019). Estudos futuros trariam maior contribuição ampliando a investigação sobre resultados e impactos destas ações na performance de inovação de startups e novas empresas de base tecnológica.

\section{AGRADECIMENTOS}

Agradecemos ao apoio institucional e de fomento à Fundação de Apoio à Pesquisa do Distrito Federal (FAP-DF).

\section{REFERÊNCIAS}

ABStartups (2019). Associação Brasileira de Startups: Mapeamento de comunidades região Centro-Oeste. Recuperado de https://abstartups.com.br/mapeamento-Centro-Oeste/

Audretsch, D. B., Cunningham, J. A., Cunningham, J. A., Kuratko, D. F., Kuratko, D. F., \& Menter, M. (2019). Entrepreneurial ecosystems: Economic, technological, and societal 
impacts. The Journal of Technology Transfer, 44(2), 313-325.

Bhawe, N., Bhawe, N., Zahra, S. A., \& Zahra, S. A. (2017). Inducing heterogeneity in local entrepreneurial ecosystems: The role of MNEs. Small Business Economics, 52(2), 437454.

BioTIC (2020). O Parque Tecnológico de Brasília. Recuperado de https://www.BioTICsa.com.br/o-parque-tecnologico-de-brasilia/

Carayannis E. G., Campbell D. F. J. (2009). "Mode 3" and "quadruple helix": toward a 21st century fractal innovation ecosystem. International Journal of Technology Management, 46(3/4), 201-234.

Carayannis, E. G., Barth, T. D., \& Campbell, D. F. (2012). The quintuple helix innovation model: Global warming as a challenge and driver for innovation. Journal of Innovation and Entrepreneurship, 1(1), 1-12.

Cidade Ocidental (2020). Parque Tecnológico Alphaville. Recuperado de http://www.cidadeocidental.go.gov.br/noticia/589-parque-tecnologico-alphavillerecebe-autoridades/

Cohen, W., \& Levinthal, D. (1990). Absorptive Capacity: A New Perspective on Learning and Innovation. Administrative Science Quarterly, 35(1), 128-152.

Díez-Vial, I., \& Montoro-Sánchez, A. (2016). How knowledge links with universities may foster innovation: The case of a science park. Technovation, (50), 41-52.

Etzkowitz, H., \& Leydesdorff, L. A. (1997). Universities and the global knowledge economy: A triple helix of university-industry-government relations. London;Washington;: Pinter.

FAP-DF (2018). Relatório de Atividades 2017. Recuperado de http://www.fap.df.gov.br/wpcontent/uploads/2018/05/Relat\%C3\%B3rio-de-Atividades-e-Gest\%C3\%A3o-2017.pdf

FAP-DF (2019). Edital de Chamamento Público $N^{\circ}$ 03/2019 - FAP-DF Animação do Ecossistema de Inovação do DF. Anexo II - Contexto e Apresentação do Edital. Recuperado de http://www.fap.df.gov.br/wp-content/uploads/2019/05/EditalEcossistema-de-inova\%C3\%A7\%C3\%A3o-Anexo-II.pdf

FAP-DF (2019). Relatório de Atividades 2018. Recuperado de http://www.fap.df.gov.br/wpcontent/uploads/2018/05/Relat\%C3\%B3rio-de-Atividades-2019.pdf

FAP-DF (2020). Relatório de Atividades 2019. Recuperado de http://www.fap.df.gov.br/wpcontent/uploads/2018/05/Relat\%C3\%B3rio-de-Atividades-2019.pdf

FAP-DF (2020). Sobre a Fundação a Fundação de Apoio à Pesquisa do Distrito Federal. Recuperado de http://www.fap.df.gov.br/sobre-a-fundacao/

GDF (2020). Edifício de governança do BioTIC - Parque Tecnológico está quase pronto. http://brasilia.df.gov.br/edificio-de-governanca-do-BioTIC-parque-tecnologico-estaquase-pronto/

Martin-Rios, C., \& Erhardt, N. (2017). Small business activity and knowledge exchange in informal interfirm networks. International Small Business Journal, 35(3), 285-305.

Monteiro, G. F. A. (2019). High-growth firms and scale-ups: A review and research agenda. RAUSP Management Journal, 54(1), 96-111. 
PCTec/UnB (2020). Sobre o Parque Cientifico e Tecnológico da UnB. Recuperado de http://www.pctec.unb.br/

Parida, V., Wincent, J. (2019). Why and how to compete through sustainability: A review and outline of trends influencing firm and network-level transformation. International Entrepreneurship and Management Journal, 15(1), 1-19.

Pereira, B., \& Farias, J., (2018). Literatura Qualificada sobre Capacidade Absortiva para Inovação em Empresas de Base Tecnológica e start-ups. Congresso Internacional de Conhecimento e Inovação - Ciki, 1(1). Recuperado de http://proceeding.ciki.ufsc.br/index.php/ciki/article/view/393

Pereira, B. A., Figlioli, A., Oliveira, D. A. F. B., \& Silva, E. R. P. (2018). Expansion and Evolution of Incubation Programs and Entrepreneurship Development in Incubators in the State of Goiás, Brazil. International Journal of Innovation, 6(1), 68-84.

Singh, S. K., \& Gaur, S. S. (2018). Entrepreneurship and innovation management in emerging economies. Management Decision, 56(1), 2-5.

Sjödin, D. (2019). Knowledge processing and ecosystem co-creation for process innovation: Managing joint knowledge processing in process innovation projects. International Entrepreneurship and Management Journal, 15(1), 135-162.

Skute, I., Zalewska-Kurek, K., Hatak, I., \& de Weerd-Nederhof, P. C. (2019). Mapping the field: A bibliometric analysis of the literature on university-industry collaborations. Journal of Technology Transfer, 44(3), 916-947.

Stam, E., \& Van de Ven, A. H. (2019). Entrepreneurial ecosystem elements. Small Business Economics, forthcoming paper, 1-24.

Whow (2020). Conheça o ecossistema de inovação do Distrito Federal. Recuperado de https://www.whow.com.br/inovacao/conheca-o-ecossistema-de-inovacao-do-distritofederal/

Wu, J., Ye, R., Ding, L., Lu, C., \& Euwema, M. (2017). From "transplant with the soil" toward the establishment of the innovation ecosystem: A case study of a leading high-tech company in China. Technological Forecasting and Social Change, 136, 222-234. 